\title{
Correction to: Integrated Metabolomics and Transcriptomics Analyses Reveal Metabolic Landscape in Neuronal Cells During JEV Infection
}

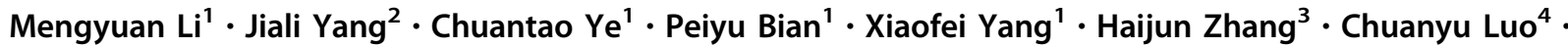 \\ Zhifeng Xue ${ }^{4} \cdot$ Yingfeng Lei $^{5}$ (D) Jianqi Lian ${ }^{1}$ (I)
}

Published online: 24 November 2021

(C) Wuhan Institute of Virology, CAS 2021

\section{Correction to: Virologica Sinica}

https://doi.org/10.1007/s12250-021-00445-0

In the original version of this article, one image in Fig. 4 was accidently duplicated during figure layout and the dilution rate was mislabeled. The correct Fig. 4 and its legend are given below:

The original article can be found online at https:// doi.org/10.1007/s12250-021-00445-0.

Jianqi Lian

lianjq@fmmu.edu.cn

$\bowtie$ Yingfeng Lei

yflei@fmmu.edu.cn

1 Department of Infectious Diseases, Tangdu Hospital, Air Force Medical University, Xi' an 710038, China

2 Key Laboratory of Resource Biology and Biotechnology in Western China, Ministry of Education, College of Life Sciences, Northwest University, Xi' an 710069, China

3 Department of Neurology, Xijing Hospital, Air Force Medical University, Xi'an 710032, China

4 Pathogenic Biology, Medical College of Yan'an University, Yan'an 716000, China

5 Department of Microbiology, School of Preclinical Medicine, Air Force Medical University, Xi' an 710032, China 
Fig. 4 PPP is indispensable for JEV replication. A Heatmap analysis of significantly changed metabolites associated with purine and pyrimidine metabolism. B-G Intervention of PPP by 6-AN significantly inhibits JEV replication in Neuro2a cell line and mouse primary neurons at $24 \mathrm{hpi}$. JEV mRNA levels in JEV-infected Neuro2a cells (B) and mouse primary neurons $(\mathbf{E})$ treated with 6-AN at 24 hpi were detected by qPCR analysis. The level of mRNA expression was normalized with $\beta$-actin. The expression levels of viral protein NS3 in JEV-infected Neuro2a cells $(\mathbf{C})$ and mouse primary neurons $(\mathbf{F})$ were detected by Western blot analysis. Plaque formation assay shows the reduction of plaque generation in JEV-infected Neuro2a cells (D) and mouse primary neurons $(\mathbf{G}) .10^{3}, 10^{4}$, $10^{5}$ and $10^{6}$ represented the dilution rate. $\mathbf{H}$ qPCR analysis of JEV mRNA level shows that anaplerosis of D-ribose 5-phosphate under 6-AN treatment condition could partially restore the viral replication. $* P<0.05$; $* * P<0.01$
A

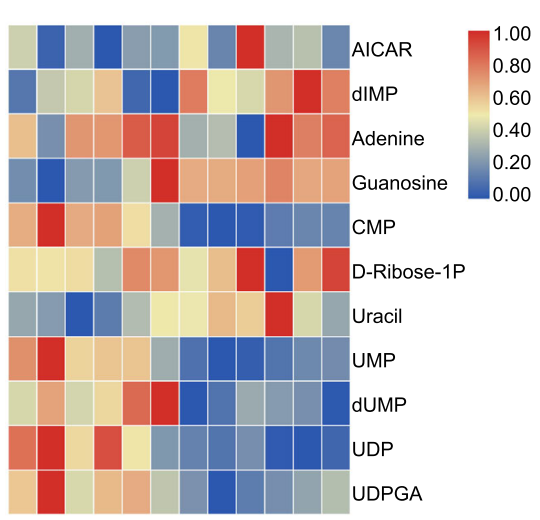

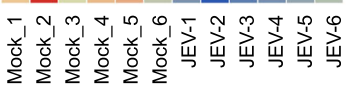

C

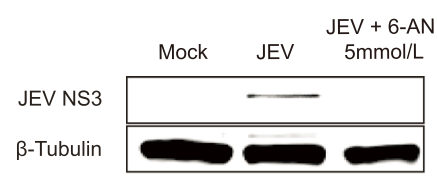

D

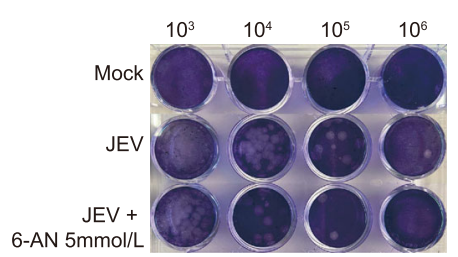

$\mathrm{F}$

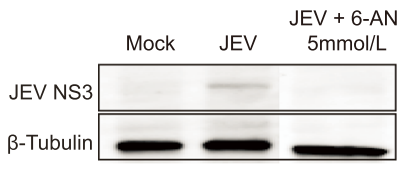

G

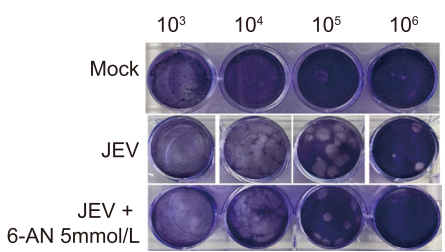

B

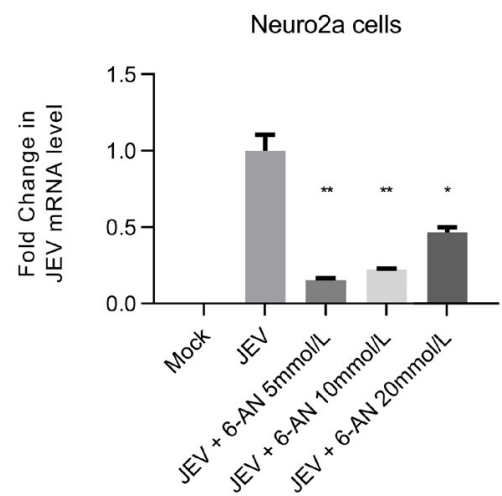

E Mouse primary neurons

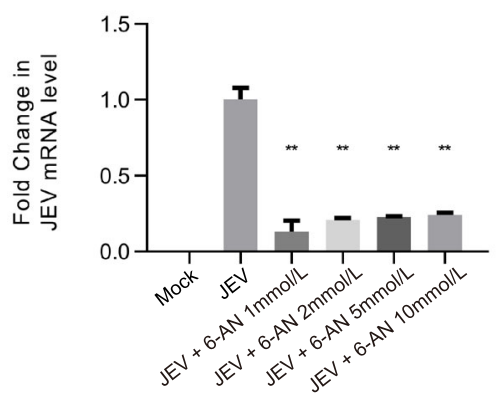

$\mathrm{H}$

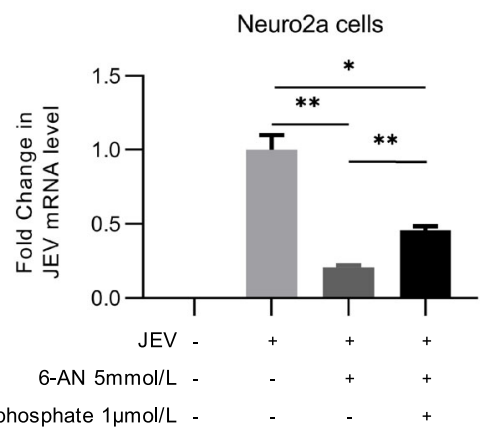

